\title{
Computational Analysis of Propulsion Performance of Modified Pitching Motion Airfoils in Laminar Flow
}

\author{
Yonghui Xie, ${ }^{1}$ Kun Lu, ${ }^{1}$ Di Zhang, ${ }^{2}$ and Gongnan Xie ${ }^{3}$ \\ ${ }^{1}$ School of Energy and Power Engineering, Xian Jiaotong University, Xian, Shaanxi 710049, China \\ ${ }^{2}$ Key Laboratory of Thermo-Fluid Science and Engineering, Ministry of Education, School of Energy and \\ Power Engineering, Xian Jiaotong University, Xian, Shaanxi 710049, China \\ ${ }^{3}$ School of Mechanical Engineering, Northwestern Polytechnical University, P.O. Box 552, Xian, Shaanxi 710072, China
}

Correspondence should be addressed to Yonghui Xie; yhxie@mail.xjtu.edu.cn

Received 24 December 2013; Accepted 13 February 2014; Published 20 March 2014

Academic Editor: Shaoyong Lai

Copyright (C) 2014 Yonghui Xie et al. This is an open access article distributed under the Creative Commons Attribution License, which permits unrestricted use, distribution, and reproduction in any medium, provided the original work is properly cited.

The thrust generation performance of airfoils with modified pitching motion was investigated by computational fluid dynamics (CFD) modeling two-dimensional laminar flow at Reynolds number of $10^{4}$. The effect of shift distance of the pitch axis outside the chord line $(R)$, reduced frequency $(k)$, pitching amplitude $(\theta)$, pitching profile, and airfoil shape (airfoil thickness and camber) on the thrust generated and efficiency were studied. The results reveal that the increase in $R$ and $k$ leads to an enhancement in thrust generation and a decrease in propulsive efficiency. Besides, there exists an optimal range of $\theta$ for the maximum thrust and the increasing $\theta$ induces a rapid decrease in propulsive efficiency. Six adjustable parameters $(K)$ were employed to realize various nonsinusoidal pitching profiles. An increase in $K$ results in more thrust generated at the cost of decreased propulsive efficiency. The investigation of the airfoil shape effect reveals that there exists an optimal range of airfoil thickness for the best propulsion performance and that the vortex structure is strongly influenced by the airfoil thickness, while varying the camber or camber location of airfoil sections offers no benefit in thrust generation over symmetric airfoil sections.

\section{Introduction}

The oscillation of an airfoil is a common form of propulsion for many animals, such as flying animals (birds, insects) and swimming species (fish, aquatic mammals). Because of its significance in studying the fundamental mechanism of flight and design of microaerial vehicles (MAVs), flapping airfoils aerodynamics has drawn much attention in recent years. Much work has been done on oscillating airfoils to examine the effects of various elements on thrust generation and propulsive efficiency. Typical flapping motions such as plunging, pitching, and combined plunging and pitching motions are defined by Rozhdestvensky and Ryzhov [1] Detailed studies related to flapping airfoils with pure pitching motion can be found in Garrick [2], Koochesfahani [3], Sarkar and Venkatraman [4], and Xiao and Liao [5]. The general conclusion is that for the pure pitching motion, positive thrust force can be generated only at a relatively high reduced frequency $(k>8)$, and the thrust generated by pure pitching motion is too low to be useful and practical.

Flapping airfoils with pure plunging motion have been extensively investigated both numerically and experimentally by Lewin and Haj-Hariri [6], Young and Lai [7], Heathcote et al. [8], and Ashraf et al. [9]. The thrust generation of an airfoil undergoing pure plunging motion is limited because of the leading edge vortex at high reduced frequency [6]. It seems that a combined plunging and pitching motion is more attractive and much research has been conducted on such motion [9-17]. The general conclusion from these investigations is that the thrust and propulsive efficiency generated by the oscillating airfoil strongly depend on the kinematic parameters such as flapping frequency, amplitude, and phase difference between pure plunging and pitching motion. A peak propulsion efficiency is observed at a phase 


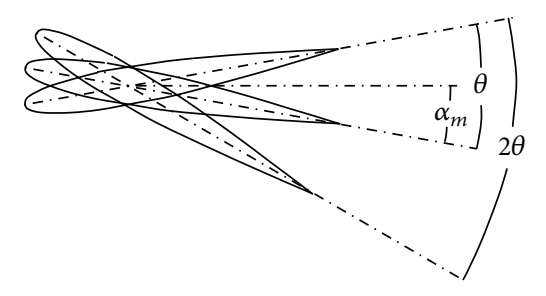

(a)

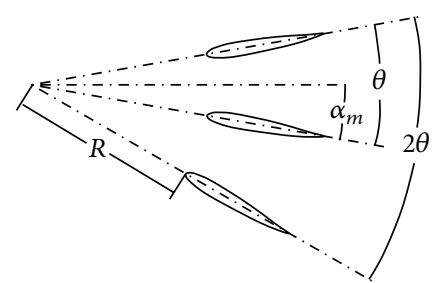

(b)

FIgURE 1: (a) Traditional pitching motion; (b) modified pitching motion with pitch axis at distance $R$ to airfoil leading edge.

angle shift of $90^{\circ}$ (pitching motion leading plunging motion by $90^{\circ}$ ) and the propulsion efficiency drops rapidly as soon as the dynamic stall occurs.

According to the studies discussed above, the application of pure pitching and plunging motions is limited due to their low thrust generation from a practical point of view, while the combined plunging and pitching motion may be limited because of its mechanical complexity. Go and Hao [18] proposed a modified pitching motion (Figure 1) and experimentally studied its propulsion characteristics in a water tunnel, and the modified pitching motion was also used by Platzer et al. [17] for MAV design. The difference between this modified pitching motion and the traditional one is the location of the pitch axis. For the traditional study of pitching airfoil aerodynamics, the pitch axis position is restricted within the chord line, while for the modified pitching motion the pitch axis lies outside the airfoil chord line and locates in front of the leading edge of the airfoil, which results in a combined plunge, pitch, and back-and-forth motion. Compared with the combined plunging and pitching motion, the modified pitching motion has an advantage in terms of mechanical simplicity and 3D motion combination effect. However, the experimental study by Go and Hao [18] is very limited because only the motion parameters of reduced frequency and pitch axis are considered and these parameters are chosen in a very small range. Besides, no numerical simulations have been conducted on the modified pitching airfoil yet.

The objective of this work is to systematically evaluate and quantify the thrust and efficiency of airfoils undergoing the proposed modified pitching motion. This modified pitching motion has the potential to increase the thrust generation through the change of motion parameters (the shift of the pitch axis by a distance $R$ outside of the chord line, reduced frequency $k$, pitching amplitude $\theta$, and pitching profile) and airfoil shape (airfoil thickness and camber). Besides, the present study also provides useful data to understand the functioning of Platzer's MAV [17].

\section{Computational Details}

2.1. Flow Solver. The CFD package CFX 13.0 with an unsteady incompressible and viscous flow solver was applied to simulate the unsteady flow field around the airfoil section. The CFX code with second-order upwind spatial discretization and a second-order-accurate backward implicit scheme for transient terms was used for all the simulations. The mass flow was evaluated such that a pressure-velocity coupling was achieved by the Rhie and Chow [19] algorithm. In the present study, all the cases of pitching airfoils were investigated at the Reynolds number of $10^{4}$ and the flow field was assumed to be laminar. The same flow solver has also been presented and employed in our previous work (Lu et al. [20]).

2.2. Dynamic Mesh Strategy. The mesh was composed of two subdomains with a sliding grid interface, and both of the two zones were meshed by a structured grid. A no-slip boundary condition was applied along the airfoil surface. The inner zone had a diameter of $12 c$ and the far-field boundary was set to at least $35 c$ from the airfoil such that its effect on the flow around the moving airfoil was negligible [21]. The dynamic mesh technique was employed for inner zone to realize the pitching motion whereas the outer zone was fixed, as employed by Wang et al. [22] and Ashraf et al. [9]. The dynamic mesh motion was controlled by a CFX expression language (CEL) subroutine developed and attached to the CFX solver. With CEL employed, the specification of the motion of nodes on boundary or subdomain regions of the mesh can be realized. The same mesh motion strategy has also been used in our previous work (Lu et al. [20]). Yang et al. [23] reported that the $\mathrm{O}$-type mesh has higher computational efficiency than the C-type mesh but yields almost the same aerodynamic forces as those resulting from the latter. Thus an $\mathrm{O}$ topology mesh was used in the inner zone. The height of the first row of cells was set at a distance to the wall of $10^{-5}$ corresponding to $y^{+}$less than one.

2.3. Motion Description. The pure sinusoidal pitching motion of airfoil section is given by

$$
\alpha(t)=\alpha_{m}+\theta \sin (2 \pi f t)
$$

where $\alpha_{m}$ is the mean angle of attack and $f$ is the pitching frequency. Sarkar and Venkatraman [4] pointed out that the pitching amplitude, mean angle of attack, reduced frequency, and pitch axis position restricted within the chord line have significant effect on thrust generation. They reveal that at a given oscillation frequency the average thrust decreases for higher values of mean angle of attack of the airfoil. As a result, $\alpha_{m}=0^{\circ}$ is chosen in this paper. 


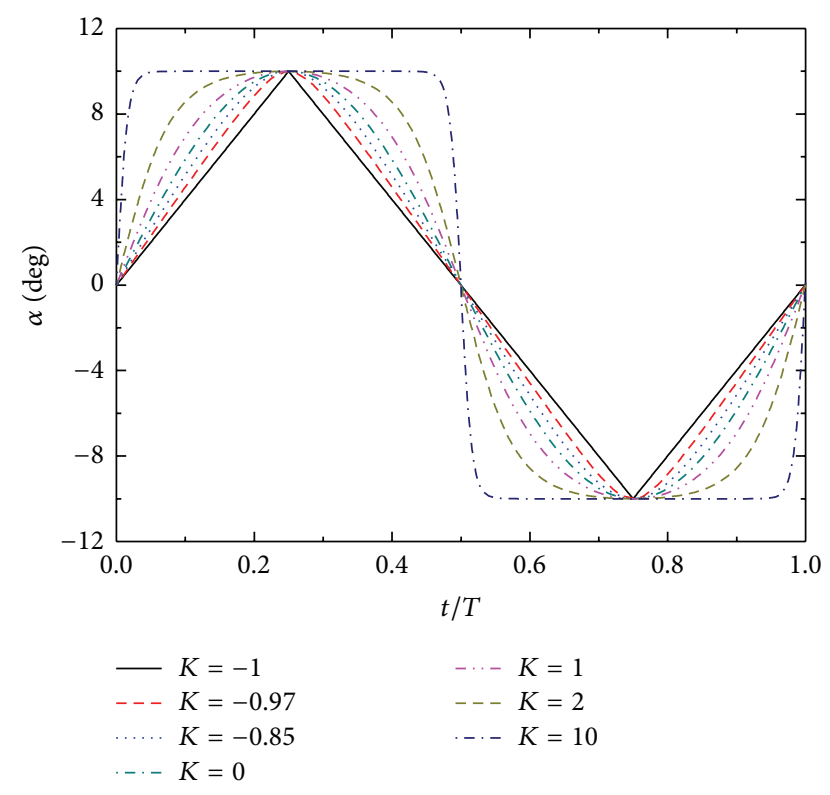

FIGURE 2: Variation of instantaneous $\alpha(t)$ profile in one period for different $K$ at $\theta=10^{\circ}$.

In the present study, the effects of pitching profile were studied with various nonsinusoidal pitching motions, which are governed by equations expressed as follows:

$$
\begin{aligned}
& \alpha(t)=\frac{\theta \sin ^{-1}[-K \sin (2 \pi f t)]}{\sin ^{-1}(-K)}, \quad-1 \leq K<0, \\
& \alpha(t)=\theta \sin (2 \pi f t), \quad K=0, \\
& \alpha(t)=\frac{\theta \tanh [K \sin (2 \pi f t)]}{\tanh K}, \quad K>0 .
\end{aligned}
$$

By tuning an adjustable parameter $K$, one can gradually change the designed pitching profile from a sawtooth wave $(K=-1)$ to a square wave $(K \rightarrow \infty)$. As a result, in effect $K$ can be viewed as a measure of how rapidly the pitching airfoil reverses direction. The sample waveform shapes for $K=-1$, $-0.97,-0.85,0,1,2$, and 10 at $\theta=10^{\circ}$ are shown in Figure 2 .

2.4. Model Validation. At first we performed a rigorous validation study to assure satisfactory independency of the force predictions with respect to both mesh and time discretizations for the present study. Three sets of mesh sizes were considered: a coarse mesh (48,000 cells and 280 nodes on the airfoil), a medium mesh (96,000 cells and 400 nodes on the airfoil), and a fine mesh (192,000 cells and 500 nodes on the airfoil). Similar observations were also made for the temporal resolution analysis. Three levels of time steps per cycle were also considered (using the medium mesh): 1200, 2400 , and 4800.

Validation tests were performed for two different typical operating points in the parametric space of pitch axis distance $R$, reduced frequency $k$, and pitching amplitude $\theta$. Both of these two cases involve dynamic stall and leading edge vortex shedding (LEVS), but at different $R, k$, and $\theta$. Results for mesh and time refinements are shown in Table 1 with time
TABLE 1: Details of grid and time steps independence study.

\begin{tabular}{lccccc}
\hline Validation & Cells & Time steps/cycle & $C_{T \text { mean }}$ & $C_{P \text { mean }}$ & $\eta$ \\
\hline \multirow{4}{*}{ Time } & \multicolumn{1}{c}{ Case $1: R=0.5 c, \theta=20^{\circ}$, and $k=3$} \\
& 96,000 & 1200 & 0.693 & 4.736 & 0.146 \\
& 96,000 & 2400 & 0.694 & 4.742 & 0.146 \\
\multirow{4}{*}{ Space } & 96,000 & 4800 & 0.697 & 4.738 & 0.147 \\
& 48,000 & 2400 & 0.697 & 4.595 & 0.152 \\
& 96,000 & 2400 & 0.694 & 4.742 & 0.146 \\
Time & 192,000 & 4800 & 0.703 & 4.763 & 0.147 \\
& 96,000 & 1200 & 0.227 & 1.134 & 0.2 \\
& 96,000 & 2400 & 0.226 & 1.135 & 0.199 \\
Space & 96,000 & 4800 & 0.227 & 1.144 & 0.198 \\
& 48,000 & 2400 & 0.231 & 1.167 & 0.198 \\
& 96,000 & 2400 & 0.226 & 1.135 & 0.199 \\
& 192,000 & 4800 & 0.230 & 1.166 & 0.197 \\
\hline \multirow{4}{*}{ Case $R=1.5 c, \theta=10^{\circ}$, and $k=2$} \\
\end{tabular}

averaged thrust coefficient, power coefficient, and efficiency considered. Figure 3 shows the time history of $C_{T}$ at $R=0.5 c$, $\theta=20^{\circ}$, and $k=3$. As can be seen, for both cases, 2400 time steps per cycle yield time-accurate predictions for both mean and instantaneous values. It is also shown that the mediummesh resolution provides satisfactory accuracy in space. For both test cases, differences between medium and fine mesh results are negligible, with variations of less than $2 \%$ on cycle averaged values, and within $4 \%$ on instantaneous peak force coefficients.

The further validation against the existing literature has been presented in our previous work (Lu et al. [20]), in which the pure plunging motion of a NACA0012 foil at $H_{0}=0.175$ and different reduced frequencies $\left(k=0-8, k=2 \pi f c / U_{\infty}\right)$ at $\mathrm{Re}=2 \times 10^{4}$ is simulated. The computational results are in close agreement with the Navier-Stokes predictions of Young and Lai [16] and experimental results by Heathcote et al. [8].

\section{Results and Discussion}

3.1. Motion Parameters. The motion parameters have a strong effect on flow dynamics through their direct effect on the airfoil inertia. To systematically evaluate and quantify how motion parameters affect the thrust performance of an airfoil undergoing the proposed modified pitching motion, a wide range of $R$ (from $0.5 c$ to $2.5 c$ ), $k$ (from 2 to 7 ), and $\theta$ (from $5^{\circ}$ to $30^{\circ}$ ) were considered. Besides, the effect of various nonsinusoidal motions on the propulsion performance of the pitching airfoil was also investigated. In this section all the computations were performed on an oscillating NACA0012 airfoil.

3.1.1. Effect of Reduced Frequency and Pitch Axis Distance. First we consider varying the reduced frequency $k$ and pitch axis distance $R$ for a given $\theta$. Figure 4 shows the variation of mean (i.e., time averaged) thrust coefficient $C_{\text {Tmean }}$ and propulsive efficiency $\eta$ with $k$ for different $R$ at $\theta=10^{\circ}$. The variations of $C_{T \text { mean }}$ with $k$ for both traditional pitching 


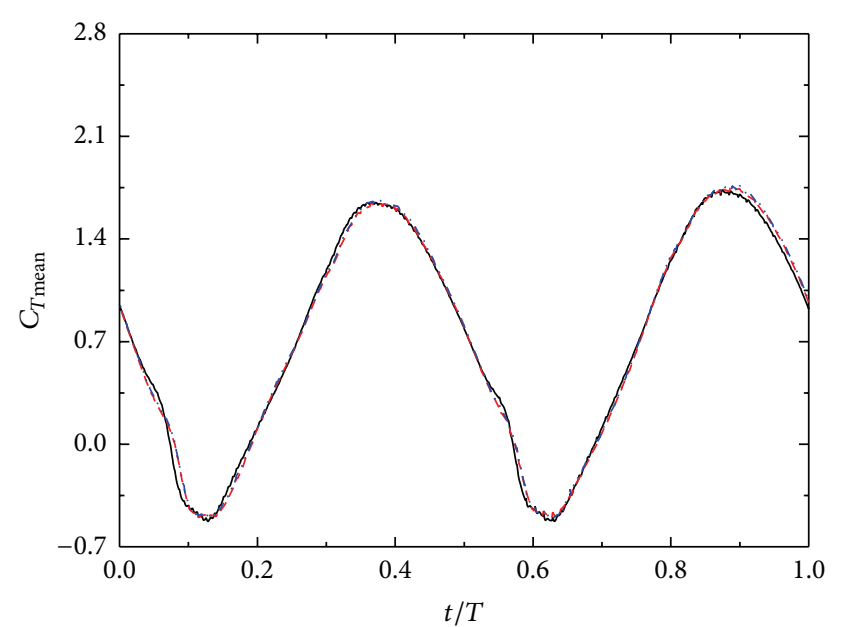

— 48,000 cell, 2400 ts/cycle

_. 96,000 cell, 2400 ts/cycle

_... 192,000 cell, 4800 ts/cycle

Figure 3: Variation of thrust coefficients with time at $R=0.5 c, \theta=$ $20^{\circ}$, and $k=3$.

motion $(R=-0.33 c)$ and modified pitching motion are shown in Figure 4. For traditional pitching motion the flow changes from drag producing to thrust producing at about $k=5$, and even in such condition the thrust generated is rather insignificant. However, the proposed modified pitching motion has great significance in thrust generation enhancement; that is, for a given $(k, \theta)$ the increasing $R$ induces much more thrust generated. As expected $C_{T \text { mean }}$ noticeably increases with $k$ and $C_{\text {Tmean }}$ appears to be a quadratic function of $k$, and this agrees with the study by Go and Hao [18].

Sarkar and Venkatraman [4] reported that the thrust generation by an oscillating airfoil mainly depends on the qualitative wake structure behind the trailing edge of the airfoil. When a pitching airfoil operates in a thrust producing regime, the wake structure behind the airfoil is opposite in sense to the Karman vortex pattern. The wake structure called "jet" gives rise to a net thrust on the airfoil. At a fixed $\theta$, an increase in $k$ and $R$ results in larger plunge amplitude and maximum pitch rate, leading to stronger reverse Von Karman vortex and averaged jet in the wake (not present here for conciseness). Thus, the increasing $R$ and $k$ lead to thrust generation enhancement. Meanwhile, the increasing $R$ and $k$ create a larger maximum effective angle of attack and bring a negative consequence; that is, the larger $R$ and higher $k$ cause more severe leading edge vortex shedding, which tends to reduce propulsive efficiency (Figure 4) [16].

Figure 5 shows the time histories of $C_{T}$ and $C_{P}$ for one flapping cycle (with $t / T=0$ corresponding to $\alpha=0^{\circ}$ and the airfoil pitching downwards) at $\theta=10^{\circ}$ considering the effects of $k$ and $R$. The comparison between the curves for the cases of $R=0.5 c, c$, and $1.5 c$ at $k=3$ reveals that the instantaneous thrust coefficient at larger $R$ is systematically larger than that at smaller $R$. Besides, the increasing $R$ induces a significant increase in maximum thrust coefficient and input power coefficient, and this can be attributed to the higher maximum pitching rate induced by the larger $R$. Similar effect of the increasing $k$ at fixed $R$ on $C_{T}$ and $C_{P}$ can also be found in Figure 5. For all the cases shown in Figure 5, the maximum $C_{T}$ occurs as the airfoil pitches towards the mean position and the minimum $C_{T}$ occurs as the airfoil pitches away from the mean position. This can be explained by the airfoil motion and the corresponding flow physics around the airfoil. The formation of reverse Von Karman vortex in the wake, which causes the thrust generation, begins as the airfoil pitches towards the mean position $(t / T=0.25-0.5$ and $0.75-1)$, and the process is almost complete when the airfoil reaches the mean position. As the airfoil pitches away from the mean position, the reverse Von Karman vortex has been shed into the wake and moves downstream. Besides, the occurrence of leading edge vortex shedding contributes to drag as it acts on a section of the airfoil surface that is facing downstream and thus causes a backward suction, leading to low level of $C_{T}$. As a result, most of the thrust is generated as the airfoil pitches towards the mean position.

3.1.2. Effect of Pitching Amplitude. The primary objective of this section is to study the effect of pitching amplitude $\theta$ on the modified pitching airfoil propulsion performance. While at fixed $k$ and $R$, changing $\theta$ affects crucial characteristics such as the maximum pitch rate and effective angle of attack profile experienced by the airfoil. Figure 6 shows the variation of $C_{T \text { mean }}$ and $\eta$ with $\theta$ for different $R$ at $k=3$. The time histories of $C_{T}$ and $C_{P}$ for one flapping cycle at $R=c$ and $k=3$ for different $\theta$ are shown in Figure 7. Figure 6 clearly shows that when a pitching airfoil operates in a thrust producing regime, there exists an optimal range of pitching amplitude for maximum thrust. At relatively small $\theta$, an increase in $\theta$ leads to more thrust generated, and the effect of increasing $\theta$ is similar to that of increasing $R$ and $k$. However, as $\theta$ reaches some degree the increase in $\theta$ brings no improvement in thrust generation while significant increase in power consumption. The vorticity fields for $\theta=5^{\circ}, 10^{\circ}$, and $15^{\circ}$ at $R=$ $c$ and $k=3$ as the airfoil pitching upward and passing though the mean position shown in Figure 8 reveal that the larger $\theta$ results in more vigorous reverse Von Karman vortex street, and we believe that this is the reason that an increase in $\theta$ can enhance the thrust performance. However, the increasing $\theta$ induces larger scales of leading edge vortices, which lead to greater fluctuation of thrust coefficient and contribute to drag as it acts on a section of the airfoil surface that is facing downstream and causes a backward suction. Besides, when the airfoil is pitching up or pitching down after passing through the mean position, the larger $\theta$ leads to higher angle of attack and pressure at the pressure surface, resulting in more drag generated. Therefore, drag producing regimes are observed between $t / T=0-0.25$ and $0.5-0.75$ at $\theta=25^{\circ}$ (Figure 7), which cause the decrease in $C_{T \text { mean }}$. However, the increasing $\theta$ always induces a significant increase in power consumption at each $R$, resulting in a rapid decrease in $\eta$.

3.1.3. Effect of Nonsinusoidal Motion. This section aims to study the effect of nonsinusoidal pitching motion of a 


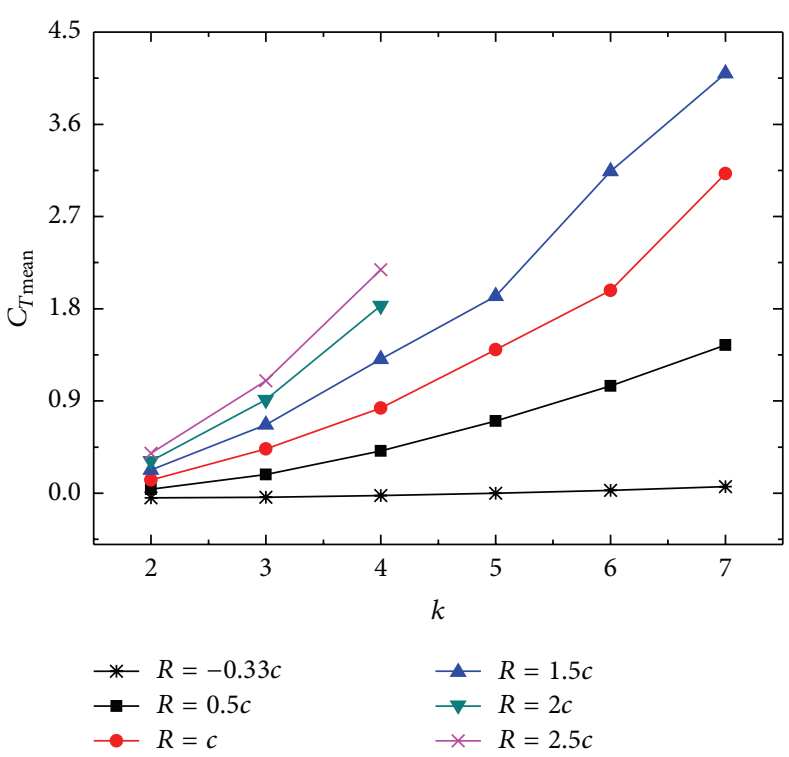

(a)

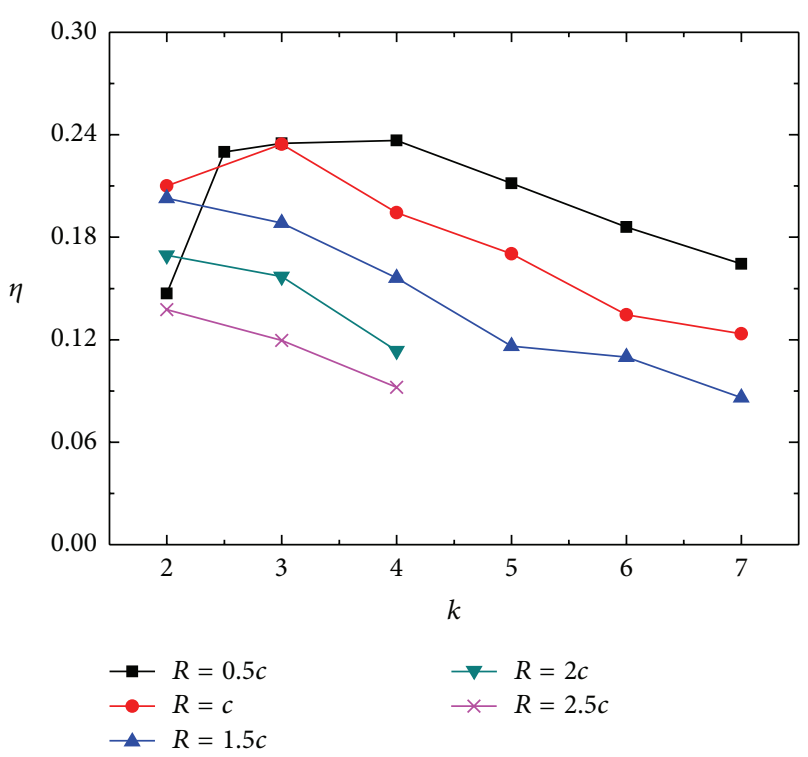

(b)

FIGURE 4: Variation of mean thrust coefficient and propulsion efficiency with $k$ at $\theta=10^{\circ}$.

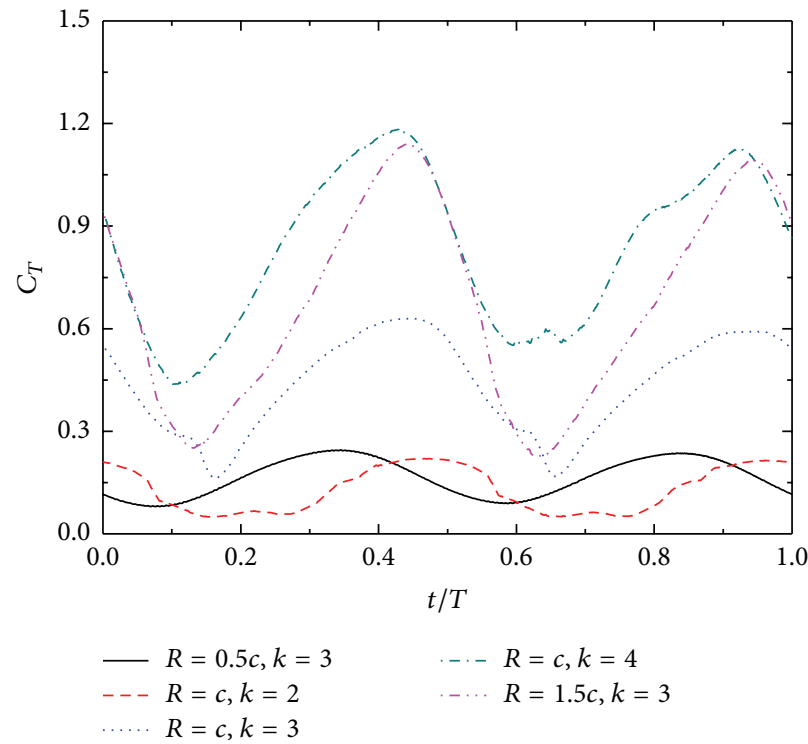

(a)

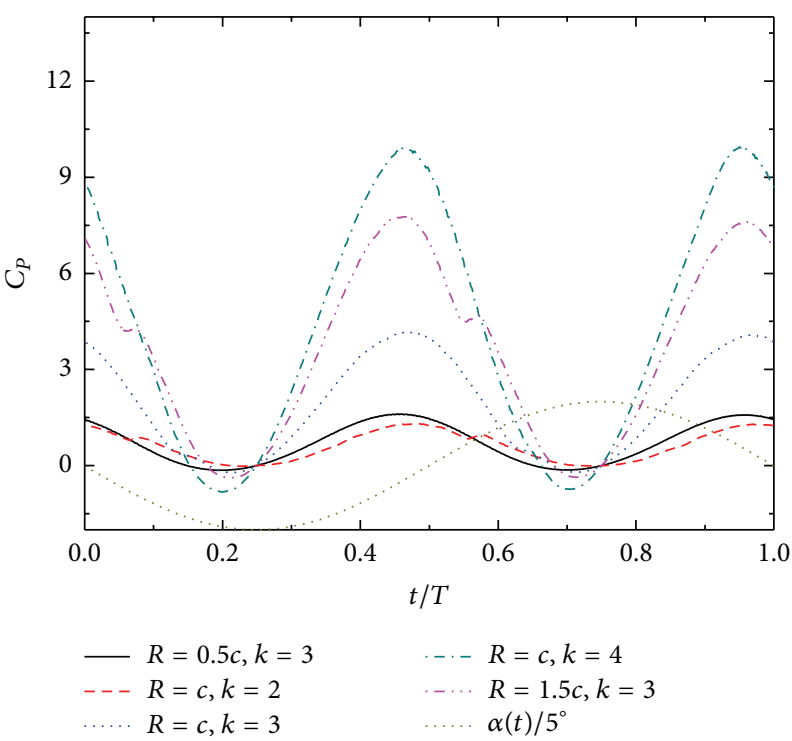

(b)

Figure 5: Variation of thrust and power coefficients with time at $k=3$ and $\theta=10^{\circ}$ for different $R$.

NACA0012 airfoil on its propulsion performance. Computations were conducted with five values of $K=-0.97$, $-0.85,0,1$, and 2 at $R=c$ and $\theta=10^{\circ}$. The effects of nonsinusoidal pitching motion on $C_{T \text { mean }}$ and $\eta$ are presented in Figure 9. Figure 10 shows the vorticity field during the upstroke for different $K$ at $t / T=0.75$ for the case of $R=$ $c, \theta=10^{\circ}$, and $k=3$. The variations of $C_{T \text { mean }}$ and $C_{P \text { mean }}$ show a similar trend; that is, both $C_{T \text { mean }}$ and $C_{P \text { mean }}$ increase with $k$. The propulsion efficiency firstly presents a flat distribution at relatively low $k$ and then steadily decreases with the increasing $k$. It is evident that the nonsinusoidal motion noticeably influences the pitching airfoil propulsion performance. Both $C_{T \text { mean }}$ and $C_{P \text { mean }}$ increase with $K$ at fixed $k$ and $\theta$, while it is the other way round in terms of $\eta$. According to (2), increasing $K$ leads to a higher maximum pitch rate, which causes stronger reverse Von Karman vortex (Figure 10) and averaged jet in the wake. As a result, the increasing $K$ leads to more thrust generated. Meanwhile, the increasing $K$ also results in a larger maximum effective angle of attack and causes more severe leading edge vortex shedding (Figure 10), which tends to reduce propulsive efficiency [16]. It is also observed that the growth of $C_{T \text { mean }}$ with $K$ is not very 

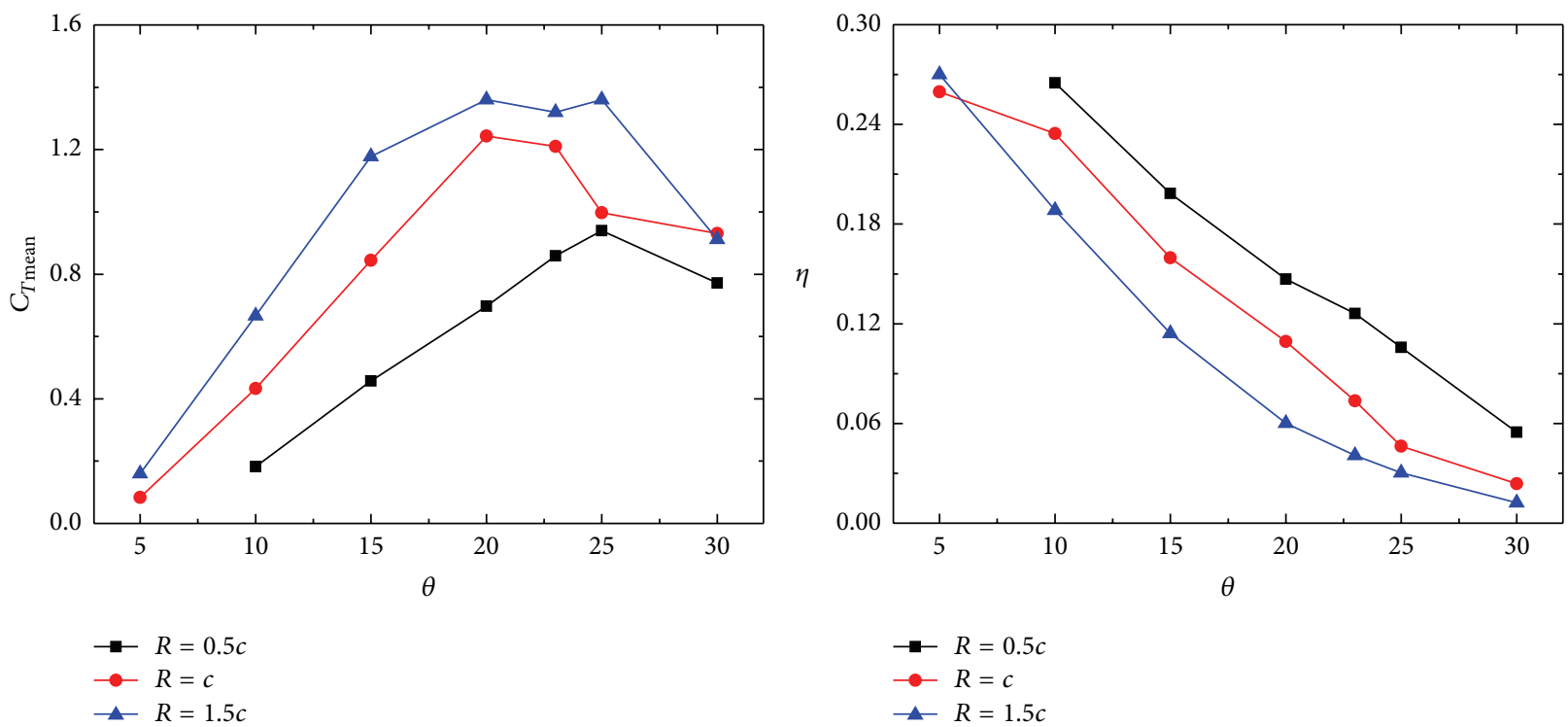

(a)

(b)

FIGURE 6: Variation of mean thrust coefficient and propulsion efficiency with amplitude at $k=3$.

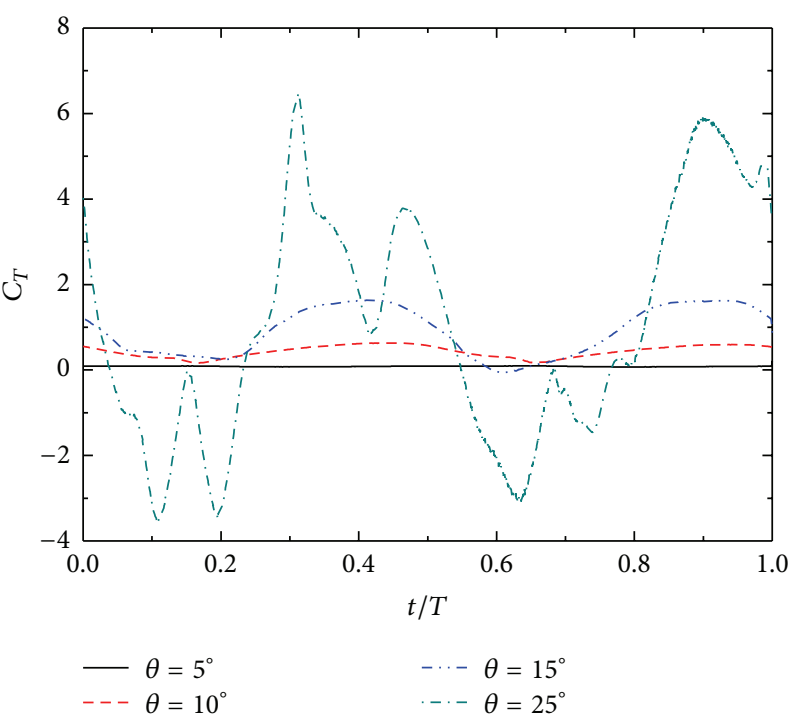

(a)

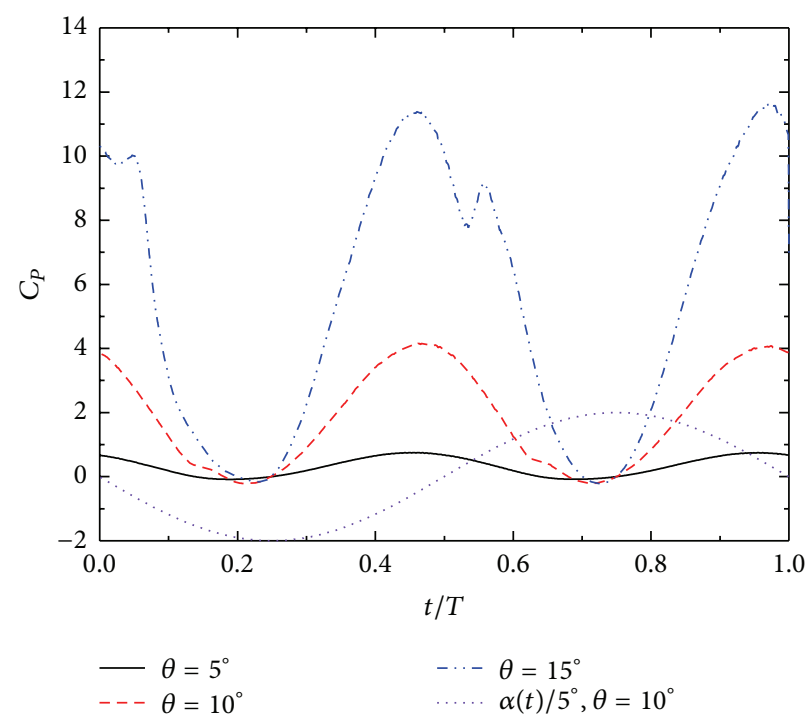

(b)

FIgURE 7: Variation of thrust and power coefficients with time at $R=c$ and $k=3$.

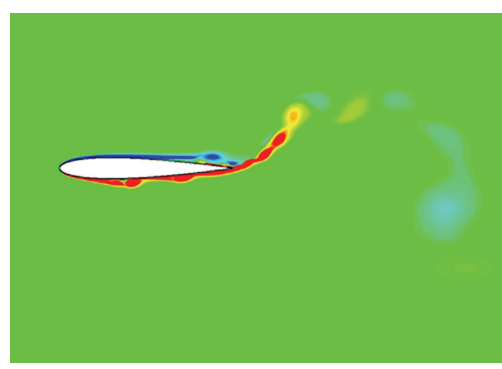

(a)

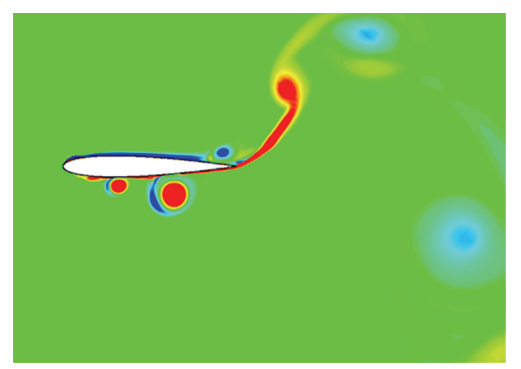

(b)

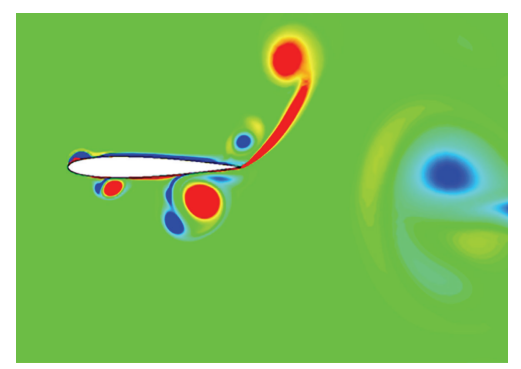

(c)

FIGURE 8: Vorticity fields at the mean position of upstroke at $R=c$ and $k=3$ for (a) $\theta=5^{\circ}$, (b) $\theta=10^{\circ}$, and (c) $\theta=15^{\circ}$. 


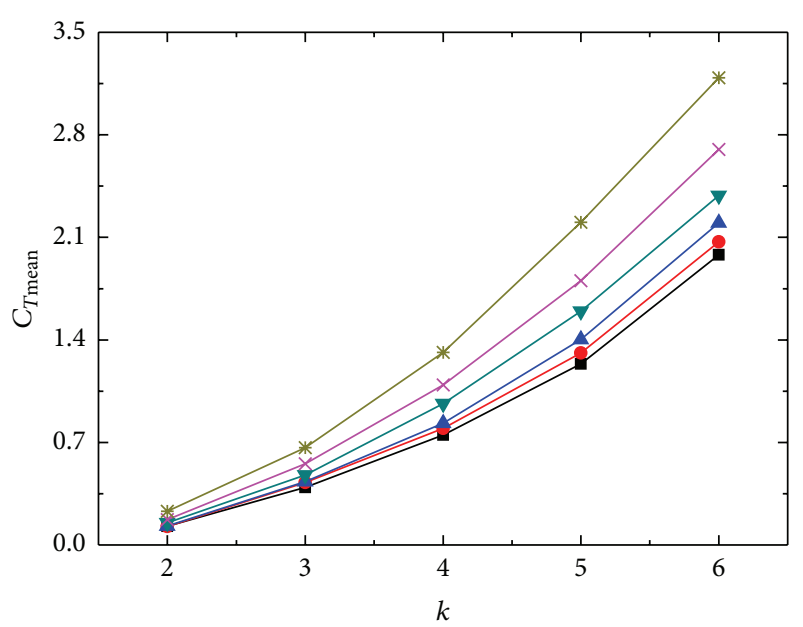

(a)

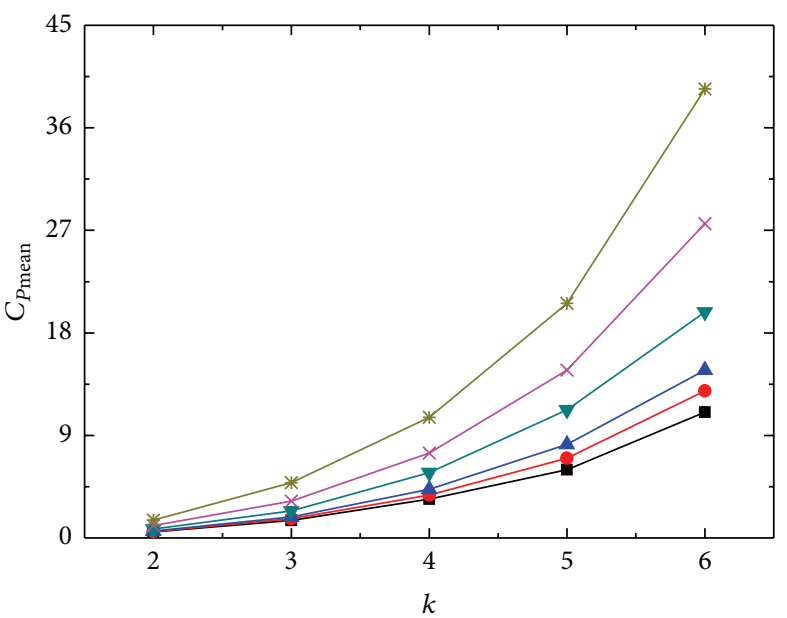

(b)

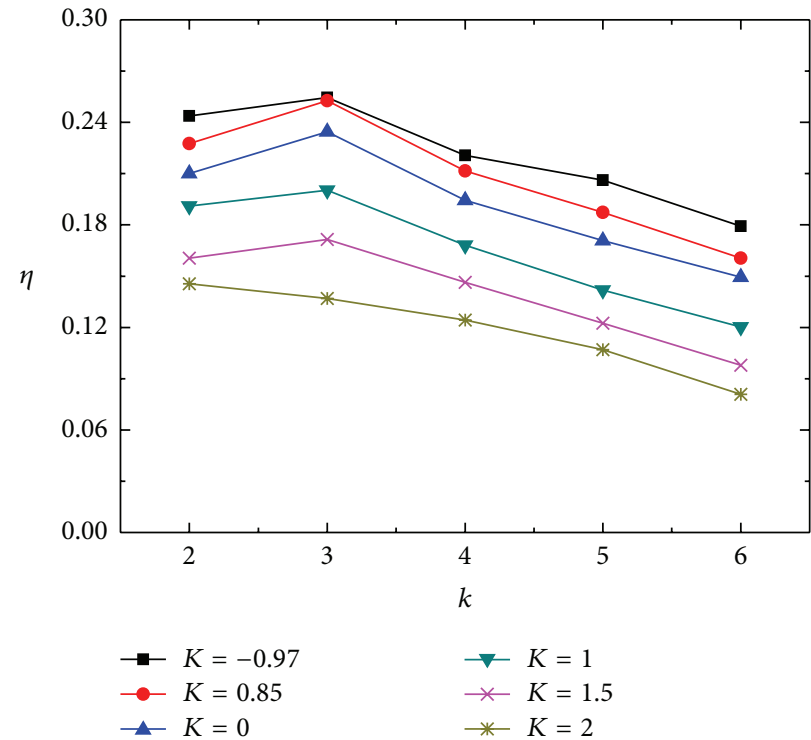

(c)

FIGURE 9: Variation of mean thrust coefficient, mean power coefficient, and propulsion efficiency with reduced frequency at $\theta=10^{\circ}$ and $R=c$.

noticeable at $K<0$ at a fixed $k$. However, while $K>0$, a faster rate of $C_{T \text { mean }}$ growth with $K$ is found. This is because the maximum pitch rate grows more and more rapidly as $K$ increases.

The effect of nonsinusoidal pitching motion on flow structure lies not only in the strength of leading edge vortex and the reverse Von Karman vortex street in the wake, but also in the formation time of the vortices. From Figure 10 we can find that the increasing $K$ induces an earlier occurrence of LEV, meaning that the LEV occurs at a lower $t / T$, while the trend for trailing edge vortex (TEV) is the other way round. Besides, as $K$ increases, a notable change in the motion of $\mathrm{TEV}$ is noticed. The vertical distance between the upper row of counterclockwise rotating vortices and the lower row of clockwise rotating vortices decreases with the increasing $K$.
To examine these results in detail, the instantaneous $C_{T}$ and $C_{P}$ in one oscillation period at $\theta=10^{\circ}$ and $R=c$ for various nonsinusoidal motions are presented in Figure 11. As expected the pitching profile noticeably affects the variation of $C_{T}$ and $C_{P}$. The increasing $K$ induces a great increase in maximum thrust coefficient and input power coefficient, and this can be attributed to the higher maximum pitch rate induced by the larger $K$. In contrast its effect on the minimum thrust coefficient and input power coefficient is quite limited. Thus $C_{T \text { mean }}$ and $C_{P \text { mean }}$ increase with $K$ at fixed $k$ and $\theta$. The larger $K$ results in the slower pitching reversal; that is, the larger $K$ induces the airfoil to oscillate at relatively high pitching amplitude for a longer time, during which the angular velocity and thrust coefficient remain at a low level (Figure 11). 


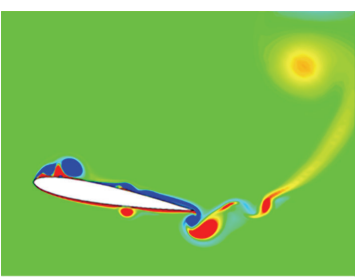

$K=-0.85$

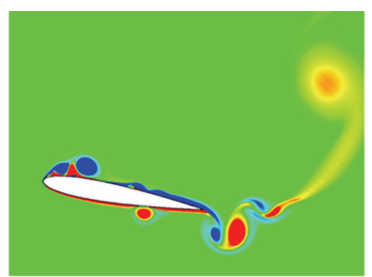

$K=0$

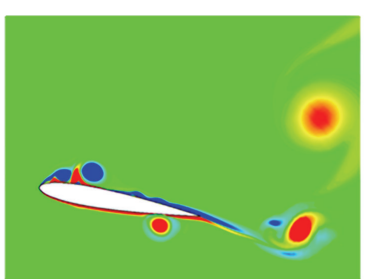

$K=1$

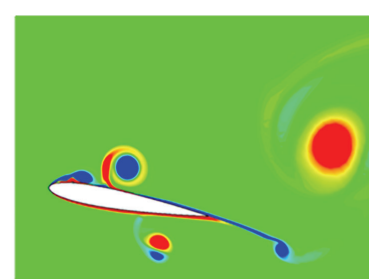

$K=2$

Figure 10: Vorticity fields during the upstroke at $t / T=0.75$ for different $K$, at $R=c, \theta=10^{\circ}$, and $k=3$.

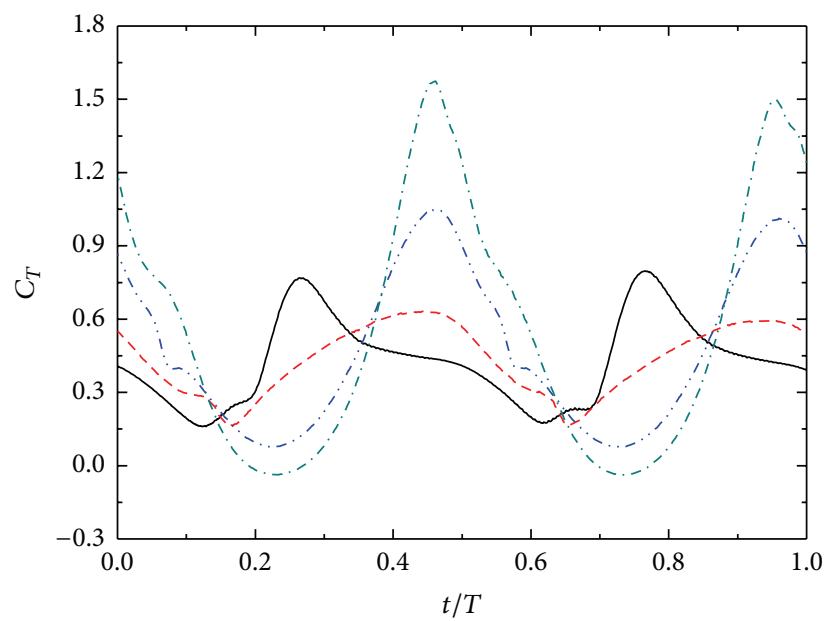

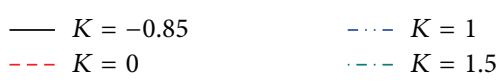

(a)

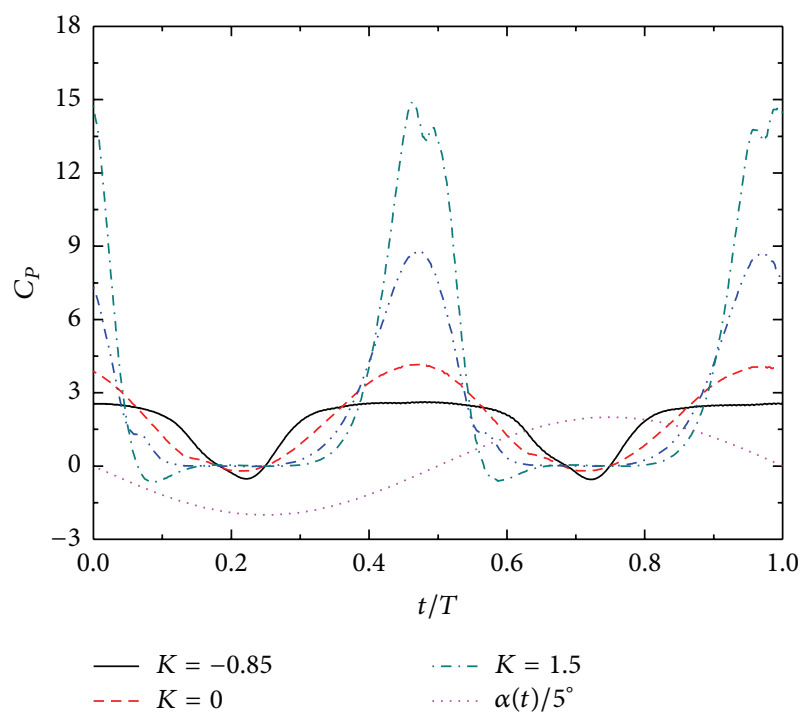

(b)

FIGURE 11: Variation of thrust and power coefficients with time at $R=c, \theta=10^{\circ}$, and $k=3$ for different $K$.

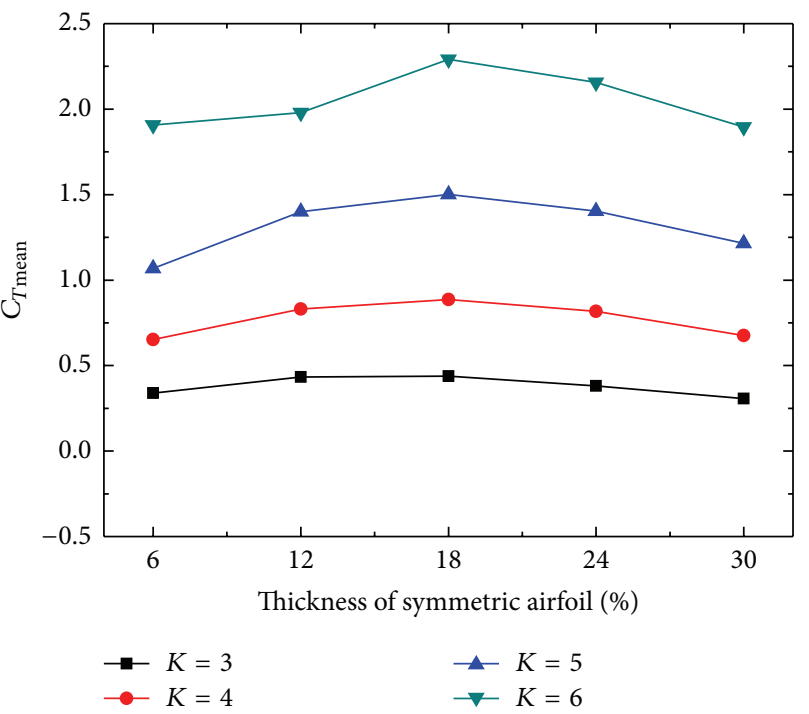

(a)

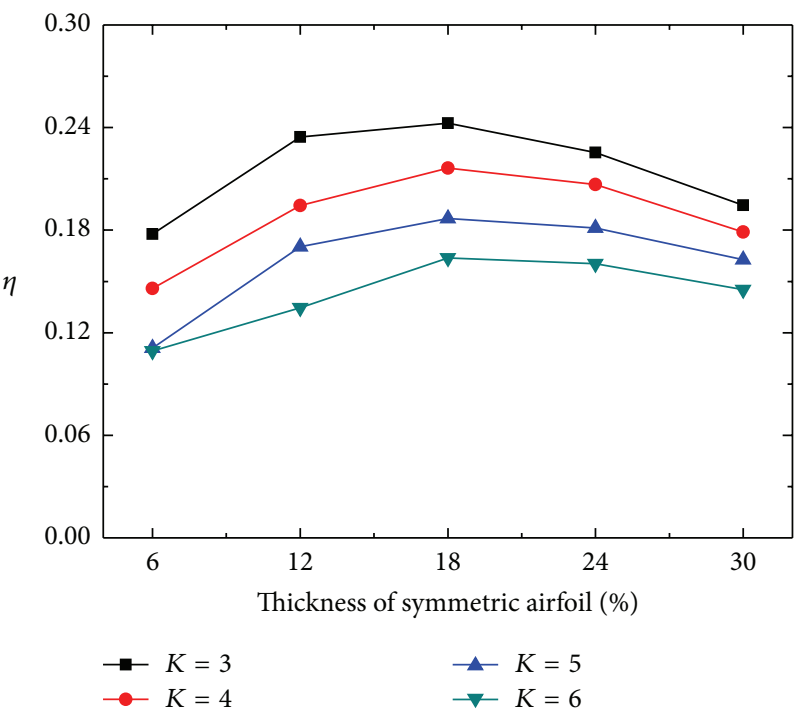

(b)

Figure 12: Variation of mean thrust coefficient and propulsion efficiency with airfoil thickness at $R=c$ and $\theta=10^{\circ}$. 


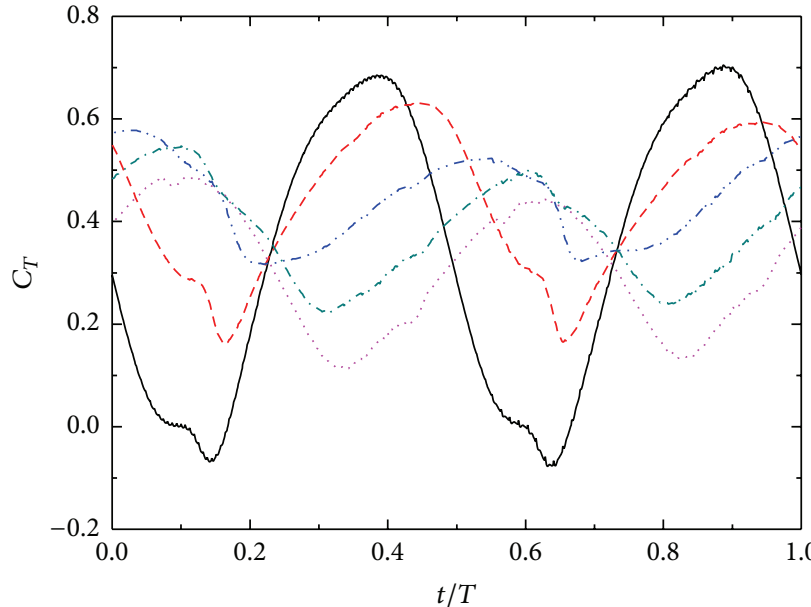

- NACA0006
- - NACA0012
-.. NACA0018

\section{... - NACA0024}

..... NACA0030

(a)

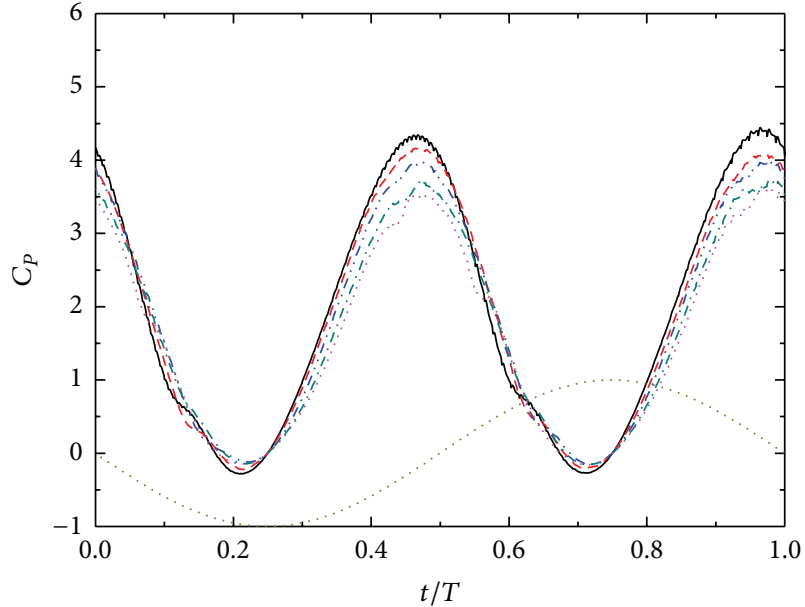

— NACA0006

- - - NACA0012

-..- NACA0018
-.- NACA0024

NACA0030

$\alpha(t) / 10^{\circ}$

(b)

FiguRE 13: Variation of thrust and power coefficients with time at $R=c, \theta=10^{\circ}$, and $k=3$ for airfoils with different thicknesses.
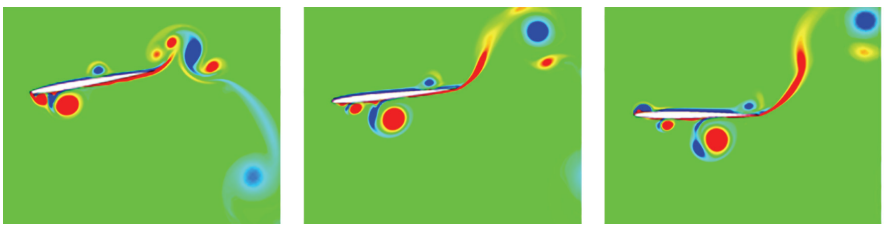

NACA0006
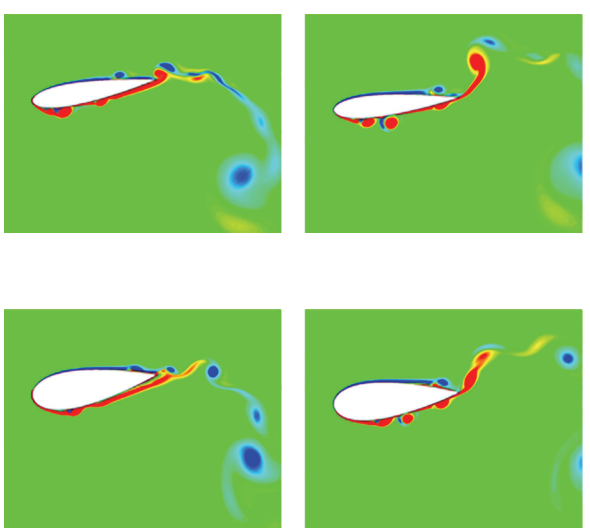

$t / T=0.25$

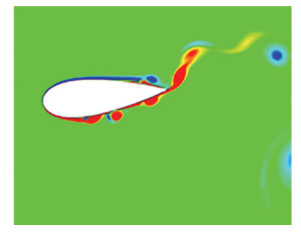

$t / T=0.4$

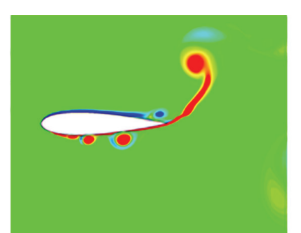

NACA0018

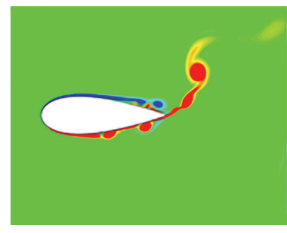

NACA0030

$t / T=0.5$
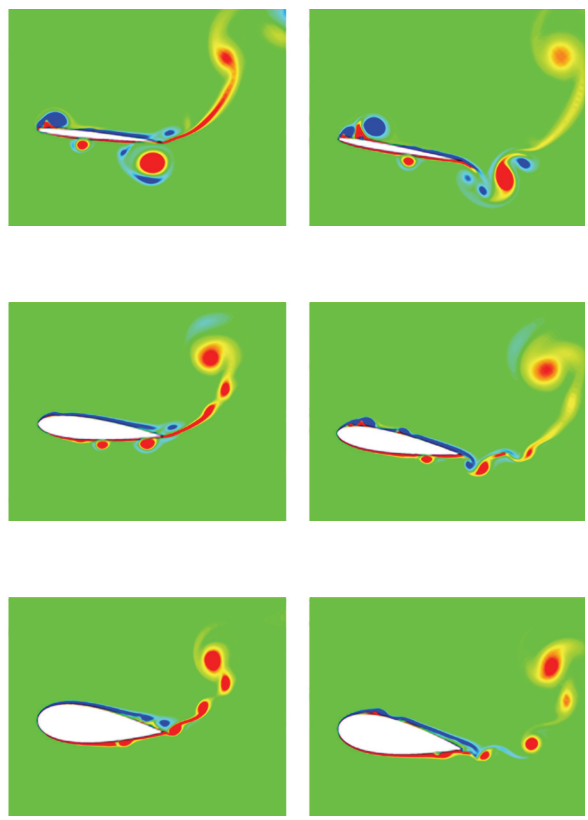

$t / T=0.6$

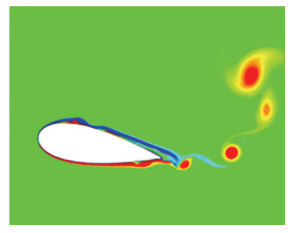

$t / T=0.75$

FIGURE 14: Vorticity fields during the upstroke at $t / T=0.25-0.75$ for NACA0006, NACA0018, and NACA0030 airfoils at $R=c, \theta=10^{\circ}$, and $k=3$.

\subsection{Airfoil Shape}

3.2.1. Effect of Airfoil Thickness. To gain some insight into the effect of airfoil thickness, simulations with five symmetric airfoils (NACA0006, NACA0012, NACA0018, NACA0024, and NACA0030) were carried out at $\theta=10^{\circ}$ and $R=c$.
The time averaged thrust coefficient $C_{T \text { mean }}$ and propulsion efficiency $\eta$ for all the tested airfoil thicknesses are presented in Figure 12. As can be seen, for each $k$ there exists an optimal range of airfoil thickness for the best propulsion performance, and the maximum thrust and efficiency are produced by the NACA0018 section. Besides, the effect of airfoil thickness on 


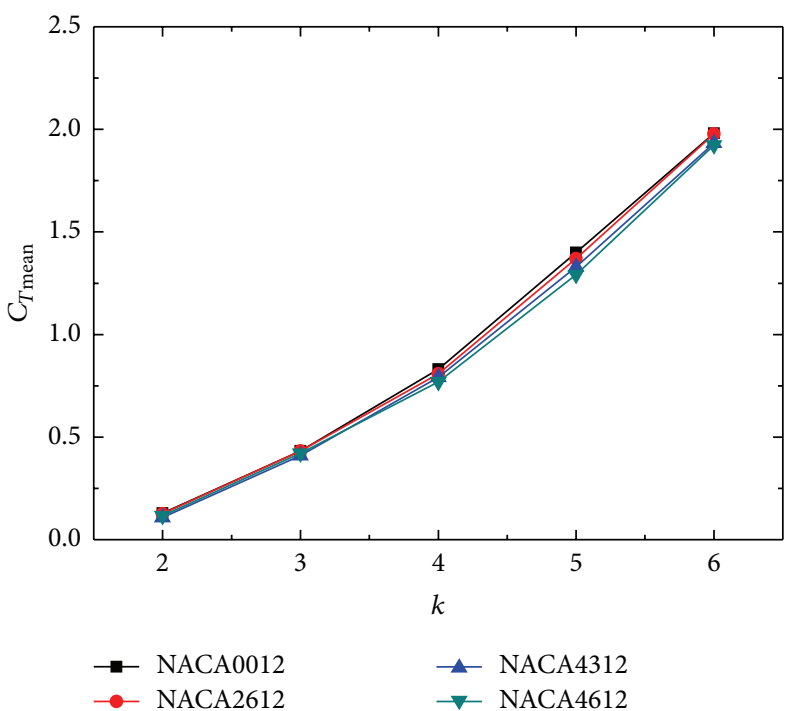

(a)

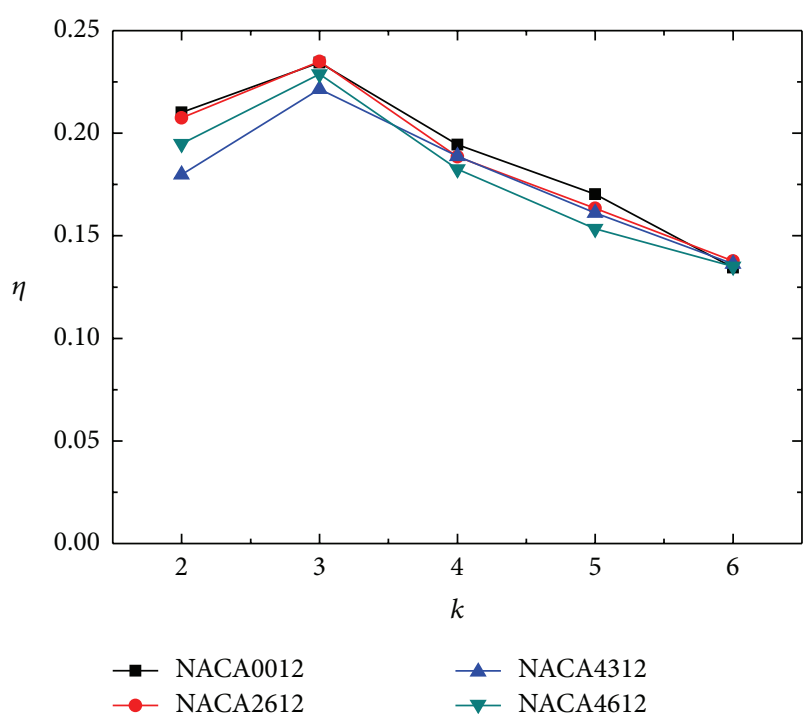

(b)

FIGURE 15: Variation of mean thrust coefficient and propulsion efficiency with reduced frequency at $R=c$ and $\theta=10^{\circ}$ for airfoils with different camber.

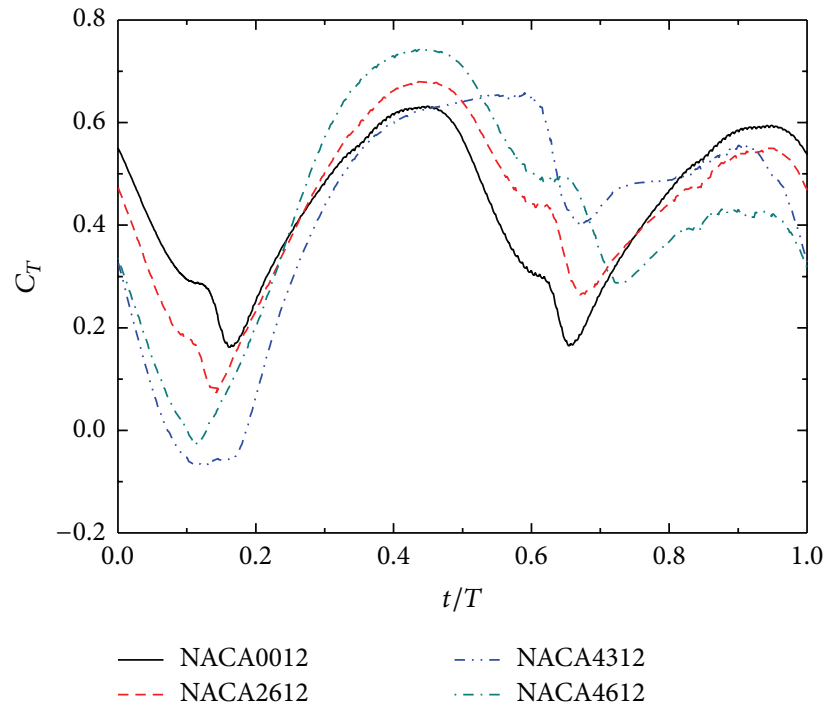

(a)

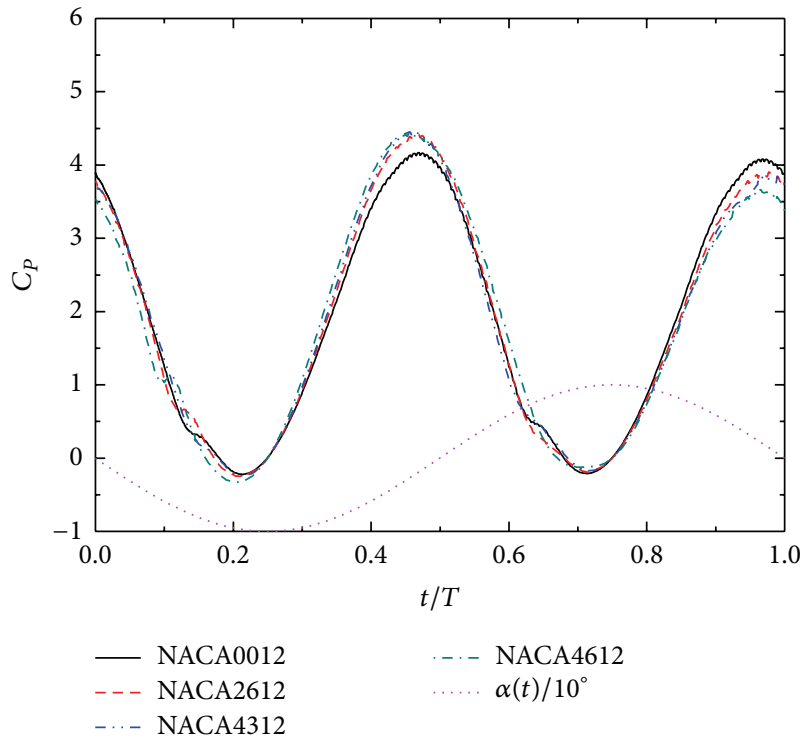

(b)

FIGURE 16: Variation of thrust and power coefficients with time at $R=c, \theta=10^{\circ}$, and $k=3$ for airfoils with different camber.

the thrust generated is more pronounced at higher $k$. Again, we find that for each airfoil $C_{T \text { mean }}$ increases with $k$, and $\eta$ tends to decrease with the increasing $k$.

Figure 13 shows time histories of $C_{T}$ and $C_{P}$ for one flapping cycle at $R=c, \theta=10^{\circ}$, and $k=3$ for airfoils with different thicknesses. We can find that the NACA0018 section generates the gentlest change in thrust, and a further increase in thickness results in a systematical decrease in instantaneous thrust coefficient. Besides, the increasing airfoil thickness induces a significant lag in the maximum thrust coefficient, meaning that the maximum thrust coefficient is obtained at a higher $t / T$. Despite the apparent differences in the variation of thrust coefficient, the resulting power coefficient varies little between the five sections, as seen in Figure 13. The greatest difference in the input power coefficient between airfoils with different thicknesses occurs at about $t / T=0.5$ and 1 , when the airfoil passes through the mean position and gets the maximum pitch rate.

In order to understand the physics behind the change in thrust performance due to increased airfoil thickness, the vorticity fields at various instances during the upstroke $(t / T=$ 


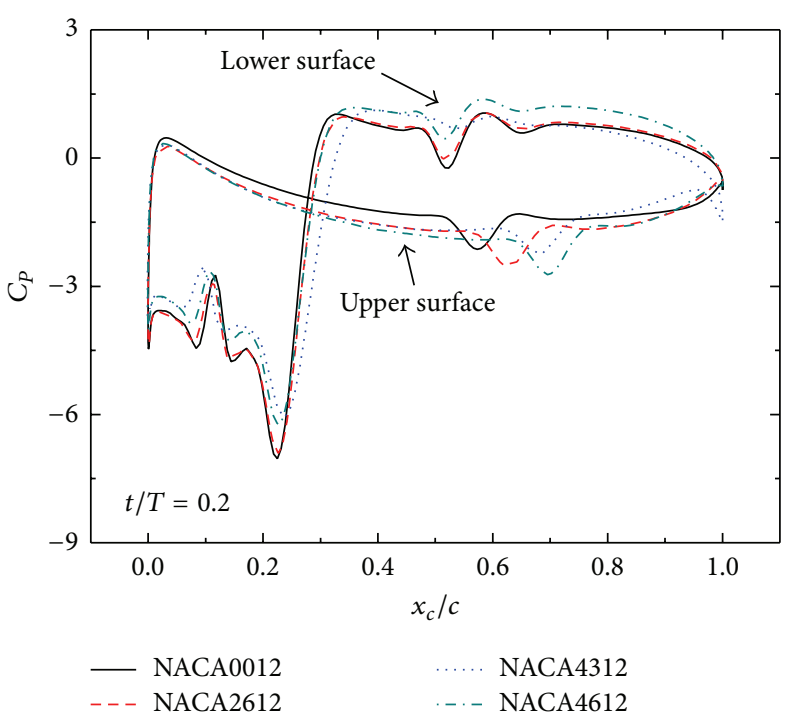

(a)

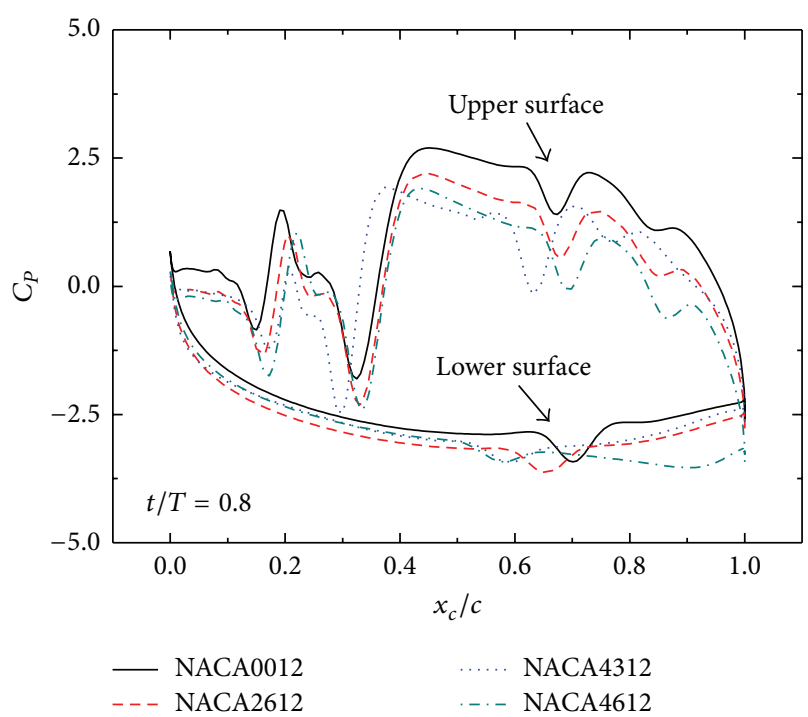

(b)

FIGURE 17: Pressure coefficient distributions of the airfoil for $R=c, \theta=10^{\circ}$, and $k=3$.

0.25-0.75) are displayed in Figure 14. Previous researchers have reported that the thrust generation by an oscillating airfoil is mainly dependent on the qualitative wake structure downstream of the trailing edge. A time averaged thrust force is generated as a reverse Von Karman vortex street occurs in the wake, as shown in Figure 14. The formation of the leading edge vortex (LEV) varies with the thickness of the airfoil section. The thin airfoil has an effectively sharper leading edge compared to the rounder leading edge of the thicker airfoils. Thus the separated flow area on the airfoil surface for NACA0006 is much larger compared with that for the NACA0018 and NACA0030 airfoils at the same instant. For NACA0006 airfoil, the large scale leading edge separation dominates pressure distribution of airfoil surface and results in the great fluctuation of thrust coefficient (Figure 13). For NACA0012 and NACA0018 airfoils, the frontal area available for the suction pressure to react upon is greater than that of NACA0006 airfoil and thus more thrust is generated by thicker airfoils, as reported by Lentink and Gerritsma [24]. However, the vorticity field for NACA0030 at $t / T=0.4-0.6$ shown in Figure 14 reveals that with a further increase in the thickness of the section, the LEV starts to form only beyond the maximum thickness point, which leads to lower thrust coefficient throughout the cycle (Figure 13). As observed in Figure 13, the power coefficient $C_{P}$ is not much altered due to varying airfoil section thickness; thus, an optimally thick airfoil section would provide more thrust and propulsion efficiency.

3.2.2. Effect of Airfoil Camber. The objective of this section is to study the camber effect on the pitching airfoil propulsion performance. Three cambered sections (NACA2612, NACA4312, and NACA4612) were chosen for the consideration of both camber and camber location. The $C_{T \text { mean }}$ and $\eta$ for the cambered sections are compared with those of the
NACA0012 (i.e., same thickness of $0.12 c$ but with no camber) in Figure 15 with sinusoidal pitching motion used at $R=c$ and $\theta=10^{\circ}$. As can be seen, differences between the $C_{T \text { mean }}$ for different airfoils at each $k$ are quite small, as well as $\eta$. Thus, in contrast to the effect of motion parameters and thickness, varying the camber or camber location of airfoil sections offers little or no benefit in thrust generation over symmetric airfoil sections.

The time histories of $C_{T}$ and $C_{P}$ in one oscillation period at $\theta=10^{\circ}$ and $R=c$ for each airfoil are presented in Figure 16 and the corresponding pressure coefficient distributions of the airfoils at $t / T=0.2$ and 0.8 are shown in Figure 17. It is observed that the airfoil camber noticeably affects the variation of $C_{T}$ and $C_{P}$. The only obvious difference in the results of a pitching cambered airfoil to that of a symmetric airfoil is that the cambered airfoil produces an asymmetric thrust coefficient during a pitching cycle. This is because the increase in thickness of upper surface and decrease in thickness on lower surface of the airfoil caused by the camber affect the pressure distribution of airfoil surface (Figure 17). Thus more thrust is generated in the upstroke of the pitching motion and approximately the same amount less is generated in the downstroke. As a result, the overall time averaged thrust remains almost the same.

\section{Summary and Conclusions}

The investigation of the thrust generation potential of airfoils with modified pitching motion has been carried out using 2D laminar flow calculations for various NACA airfoils at $\operatorname{Re}=10^{4}$. The effects of motion parameters (the shift of the pitch axis by a distance $R$ outside of the chord line, reduced frequency $k$, pitching amplitude $\theta$, and pitching profile) and airfoil shape (airfoil thickness and camber) were studied. The thrust generated and efficiency of airfoils 
undergoing the proposed modified pitching motion were systematically evaluated and quantified. The results reveal that the increase in $R$ and $k$ leads to more thrust generated. However, the trend for propulsive efficiency $\eta$ is the other way round. It is also observed that when a pitching airfoil operates in a thrust producing regime, there exists an optimal range of pitching amplitude for maximum thrust. However, the increasing $\theta$ always induces a rapid decrease in $\eta$. To realize various nonsinusoidal pitching motions of airfoil, six adjustable parameters $K=-0.97,-0.85,0,1,1.5$, and 2 were employed, among which $K=0$ corresponds to a pure sinusoidal motion. An increase in $K$ results in a better thrust generation performance at the cost of a decrease in $\eta$, especially for $K>0$.

Then the effect of airfoil shape on modified pitching airfoil propulsion performance was investigated with varying airfoil thickness and camber considered. The thickness study was performed on 2D NACA symmetric airfoils with $6 \%$, $12 \%, 18 \%, 24 \%$, and $30 \%$ thick sections. It is found that for each $k$ there exists an optimal range of airfoil thickness for the best propulsion performance, and the maximum thrust and efficiency are produced by the NACA0018 section. Besides, the vortex structure is strongly influenced by the airfoil thickness. The study of camber effect indicates that varying the camber or camber location of airfoil sections offers no benefit in thrust generation over symmetric airfoil sections.

\section{Nomenclature}

$\begin{array}{ll}c: & \text { Airfoil chord length } \\ C_{D}: & \text { Drag coefficient } \\ C_{M}: & \text { Moment coefficient } \\ C_{P}: & \text { Power coefficient } \\ C_{P \text { mean }}: & \text { Time averaged power coefficient } \\ & C_{P \text { mean }}=-(1 / T) \int_{t}^{t+T}\left[C_{D}(t) \dot{x}(t)+\right. \\ & \left.C_{L}(t) \dot{y}(t)+C_{M}(t) \dot{\alpha}(t)\right] d t / U_{\infty} \\ C_{T}: & \text { Thrust coefficient } \\ C_{T \text { mean }}: & \text { Time averaged thrust coefficient } \\ & C_{T \text { mean }}=-(1 / T) \int_{t}^{t+T} C_{D}(t) d t \\ k: & \text { Reduced frequency }\left(k=2 \pi f c / U_{\infty}\right) \\ K: & \text { Parameter used to realize various } \\ & \text { nonsinusoidal pitching profiles } \\ \text { Re: } & \text { Reynolds number } \\ T: & \text { Period of oscillation } \\ t: & \text { Physical time } \\ U_{\infty}: & \text { Freestream velocity } \\ x: & \text { Back-and-forth position of airfoil } \\ x_{c}: & \text { Chordwise position } \\ y: & \text { Plunge position of airfoil } \\ \alpha: & \text { Instantaneous angle of attack } \\ \theta: & \text { Amplitude of pitching oscillation } \\ \eta: & \text { Propulsive efficiency }\left(C_{T \text { mean }} / C_{P \text { mean }}\right) .\end{array}$

\section{Conflict of Interests}

The authors declare that there is no conflict of interests regarding the publication of this paper.

\section{References}

[1] K. V. Rozhdestvensky and V. A. Ryzhov, "Aerohydrodynamics of flapping-wing propulsors," Progress in Aerospace Sciences, vol. 39, no. 8, pp. 585-633, 2003.

[2] I. E. Garrick, "Propulsion of a flapping and oscillating airfoil," NACA TR 567, 1937.

[3] M. M. Koochesfahani, "Vortical patterns in the wake of an oscillating airfoil," AIAA Journal, vol. 27, no. 9, pp. 1200-1205, 1989.

[4] S. Sarkar and K. Venkatraman, "Numerical simulation of thrust generating flow past a pitching airfoil," Computers and Fluids, vol. 35, no. 1, pp. 16-42, 2006.

[5] Q. Xiao and W. Liao, "Numerical study of asymmetric effect on a pitching foil," International Journal of Modern Physics C, vol. 20, no. 10, pp. 1663-1680, 2009.

[6] G. C. Lewin and H. Haj-Hariri, "Modelling thrust generation of a two-dimensional heaving airfoil in a viscous flow," Journal of Fluid Mechanics, no. 492, pp. 339-362, 2003.

[7] J. Young and J. C. S. Lai, "Oscillation frequency and amplitude effects on the wake of a plunging airfoil," AIAA Journal, vol. 42, no. 10, pp. 2042-2052, 2004.

[8] S. Heathcote, Z. Wang, and I. Gursul, "Effect of spanwise flexibility on flapping wing propulsion," Journal of Fluids and Structures, vol. 24, no. 2, pp. 183-199, 2008.

[9] M. A. Ashraf, J. Young, and J. C. S. Lai, "Reynolds number, thickness and camber effects on flapping airfoil propulsion," Journal of Fluids and Structures, vol. 27, no. 2, pp. 145-160, 2011.

[10] I. H. Tuncer and M. F. Platzer, "Thrust generation due to airfoil flapping," AIAA Journal, vol. 34, no. 2, pp. 324-331, 1996.

[11] I. H. Tuncer, R. Walz, and M. F. Platzer, "A computational study on the dynamic stall of a flapping airfoil," in Proceedings of the 16th Applied Aerodynamics Conference, Albuquerque, NM, USA, 1998 .

[12] J. M. Anderson, K. Streitlien, D. S. Barrett, and M. S. Triantafyllou, "Oscillating foils of high propulsive efficiency," Journal of Fluid Mechanics, vol. 360, pp. 41-72, 1998.

[13] K. Isogai, Y. Shinmoto, and Y. Watanabe, "Effects of dynamic stall on propulsive efficiency and thrust of flapping airfoil," AIAA Journal, vol. 37, no. 10, pp. 1145-1151, 1999.

[14] R. Ramamurti and W. Sandberg, "Simulation of flow about flapping airfoils using finite element incompressible flow solver," AIAA Journal, vol. 39, no. 2, pp. 253-258, 2001.

[15] J. M. Miao and M. H. Ho, "Effect of flexure on aerodynamic propulsive efficiency of flapping flexible airfoil," Journal of Fluids and Structures, vol. 22, no. 3, pp. 401-419, 2006.

[16] J. Young and J. C. S. Lai, "Mechanisms influencing the efficiency of oscillating airfoil propulsion," AIAA Journal, vol. 45, no. 7, pp. 1695-1702, 2007.

[17] M. F. Platzer, K. D. Jones, J. Young, and J. C. S. Lai, "Flappingwing aerodynamics: progress and challenges," AIAA Journal, vol. 46, no. 9, pp. 2136-2149, 2008.

[18] T. H. Go and W. Hao, "Investigation on propulsion of flapping wing with modified pitch motion," Aircraft Engineering and Aerospace Technology, vol. 82, no. 4, pp. 217-224, 2010.

[19] C. M. Rhie and W. L. Chow, "Numerical study of the turbulent flow past an airfoil with trailing edge separation," AIAA Journal, vol. 21, no. 11, pp. 1525-1532, 1983.

[20] K. Lu, Y. H. Xie, and D. Zhang, "Numerical study of large amplitude, nonsinusoidal motion and camber effects on pitching airfoil propulsion," Journal of Fluids and Structures, vol. 36, pp. 184-194, 2013. 
[21] F. M. Bos, D. Lentink, B. W. Van oudheusden, and H. Bijl, "Influence of wing kinematics on aerodynamic performance in hovering insect flight," Journal of Fluid Mechanics, vol. 594, pp. 341-368, 2008.

[22] S. Wang, D. B. Ingham, L. Ma, M. Pourkashanian, and Z. Tao, "Numerical investigations on dynamic stall of low Reynolds number flow around oscillating airfoils," Computers and Fluids, vol. 39, no. 9, pp. 1529-1541, 2010.

[23] S. Yang, S. Luo, F. Liu, and H. Tsai, "Subsonic flow over unstalled pitching airfoil computed by Euler method," in Proceedings of the 36th AIAA Fluid Dynamics Conference and Exhibit, pp. 2744-2759, San Francisco, Calif, USA, June 2006.

[24] D. Lentink and M. I. Gerritsma, "Influence of airfoil shape on performance in insect flight," in Proceedings of the 33rd AIAA Fluid Dynamics Conference and Exhibit, Orlando, Fla, USA, 2003. 


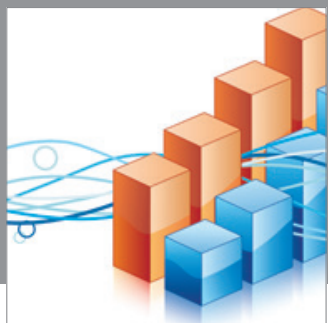

Advances in

Operations Research

mansans

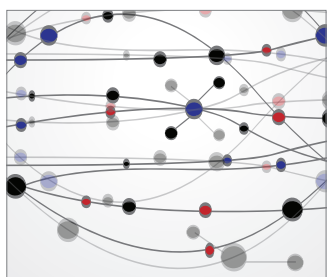

The Scientific World Journal
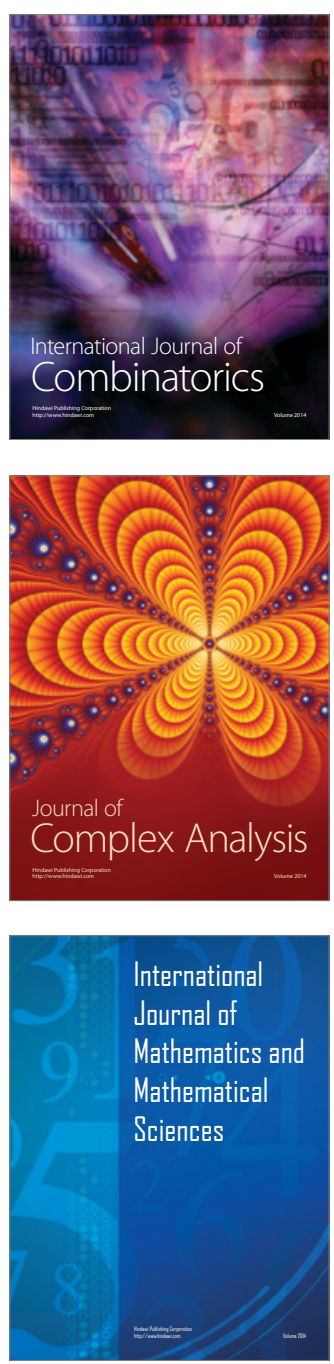
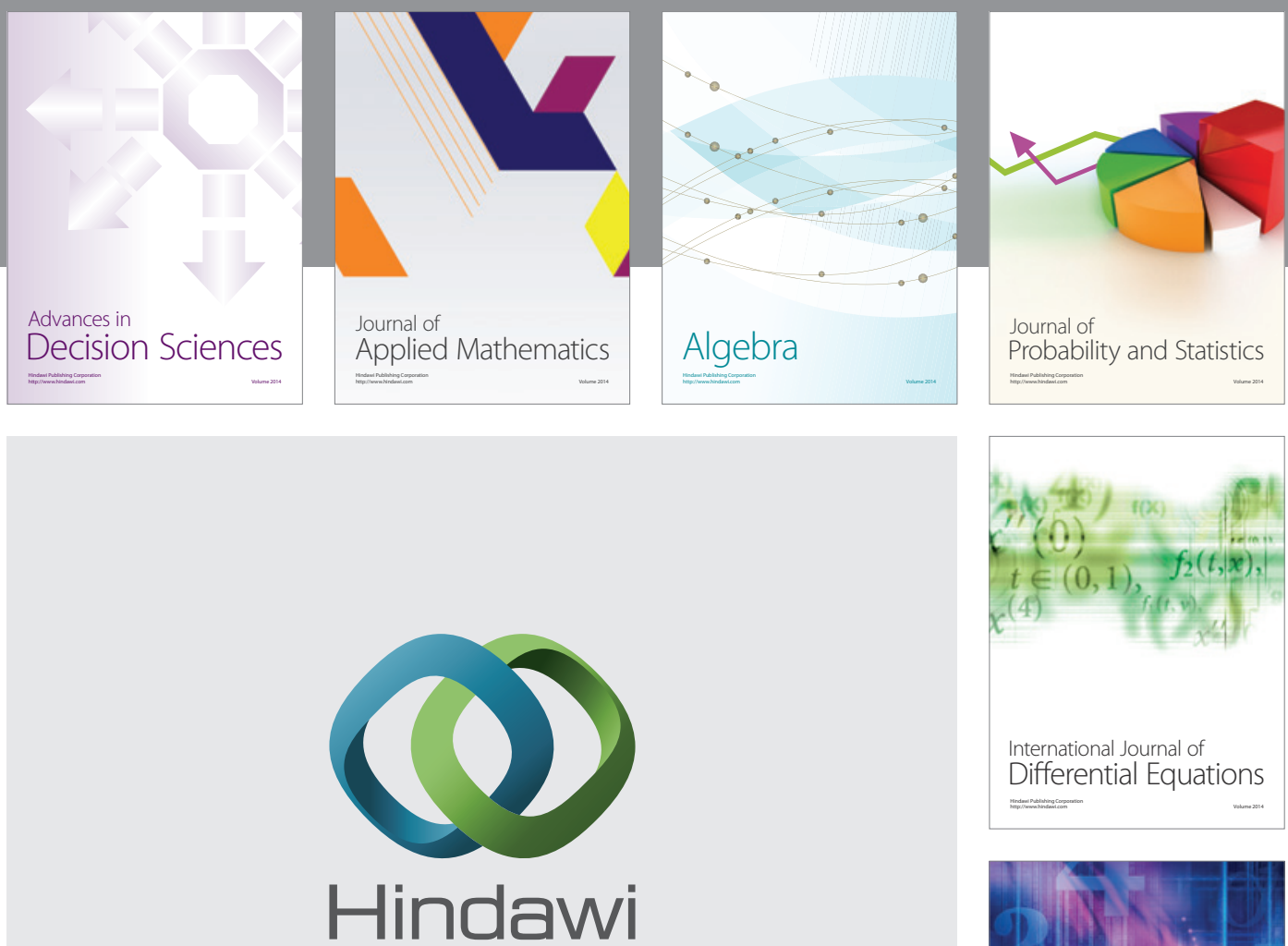

Submit your manuscripts at http://www.hindawi.com
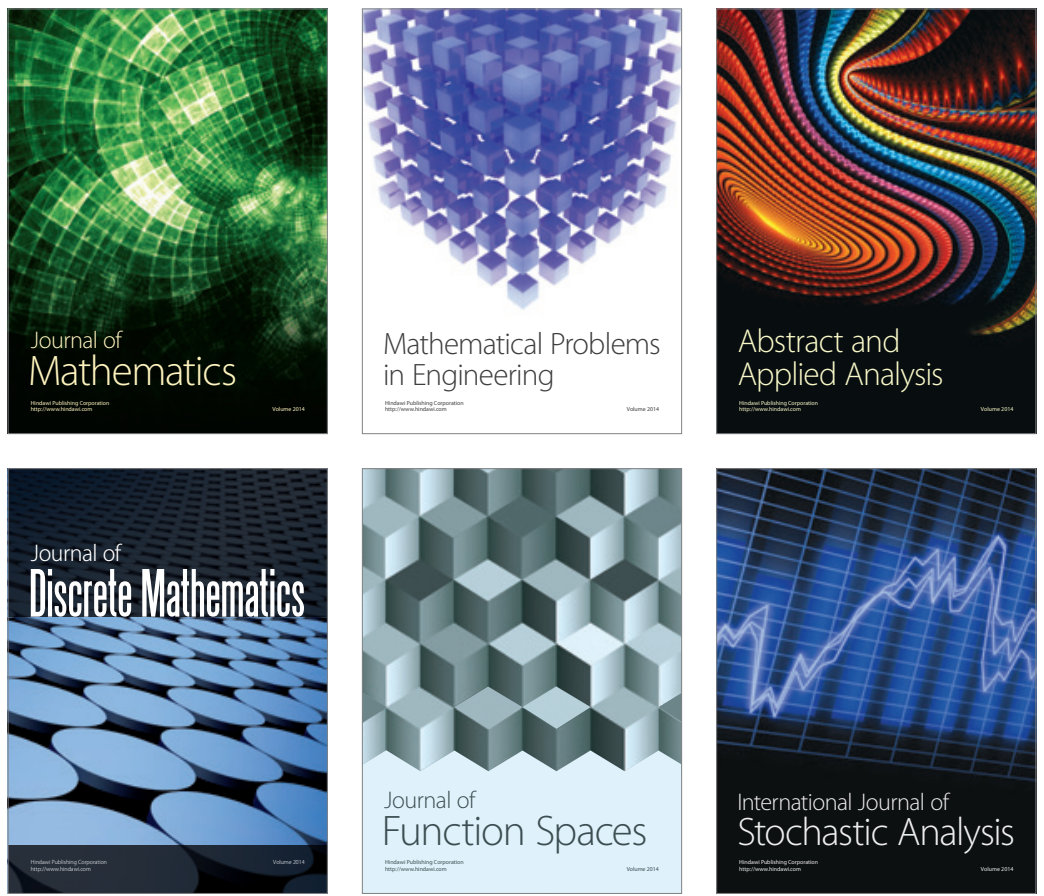

Journal of

Function Spaces

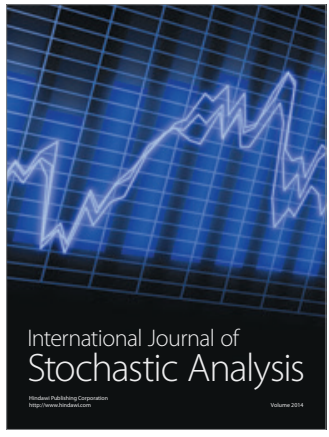

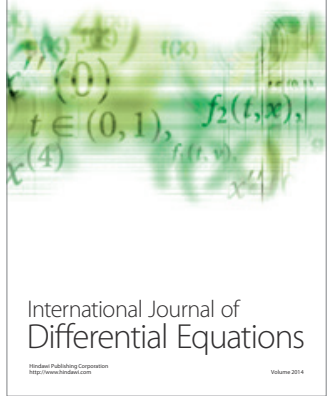
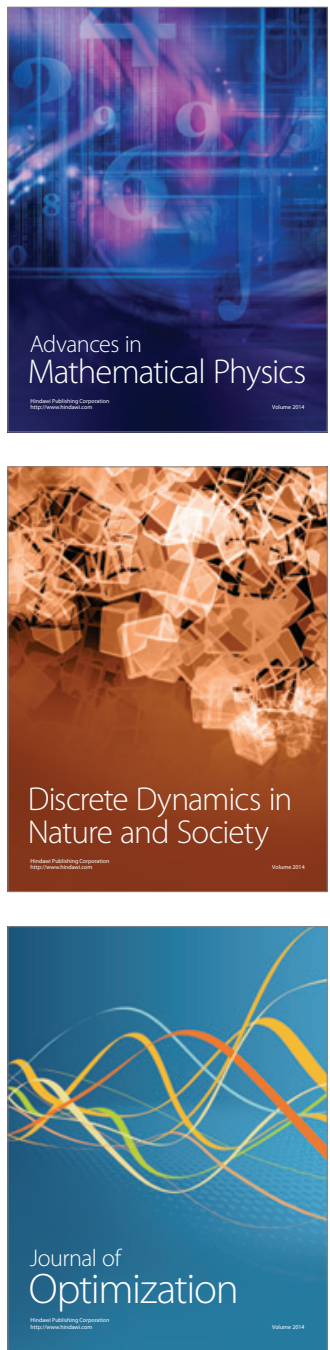